\title{
Strong paraconsistency by separating composition and decomposition in classical logic ${ }^{\star}$
}

\author{
Peter Verdée ${ }^{\star \star}$ \\ Centre for Logic and Philosophy of Science \\ Ghent University, Belgium \\ Peter.Verdee@UGent. be
}

\begin{abstract}
In this paper I elaborate a proof system that is able to prove all classical first order logic consequences of consistent premise sets, without proving trivial consequences of inconsistent premises (as in $A, \neg A \vdash B$ ). Essentially this result is obtained by formally distinguishing consequences that are the result of merely decomposing the premises into their subformulas from consequences that may be the result of also composing 'new', more complex formulas. I require that, whenever 'new' formulas are derived, they are to be preceded by a special +-symbol and these +-preceded formulas are not to be decomposed. By doing this, the proofs are separated into a decomposition phase followed by a composition phase. The proofs are recursive, axiomatizable and, as they do not trivialize inconsistent premise sets, they define a very strong non-transitive paraconsistent logic, for which I also provide an adequate semantics.
\end{abstract}

\section{Introduction}

Let a rule be a metalinguistic expression of the form "from $A_{1}, \ldots, A_{n-1}$ and $A_{n}$ derive $B$, provided condition". Instances of $A_{1}, \ldots A_{n}$ will be called the local premises of (an application of) the rule and an instance of $B$ will be called the conclusion of (an application of) the rule. In usual natural deduction proof systems for classical logic, one is able to distinguish between what I shall call compositional and decompositional rules. Compositional rules are rules of which the conclusion nor its negation occurs as a subformula in the local premises. Decompositional rules are rules that are not compositional. Examples of typical compositional rules include Addition (from ' $A$ ' derive ' $A \vee B$ '), Adjunction (from ' $A$ ' and ' $B$ ' derive ' $A \wedge B$ '), Existential Generalization (from ' $A(\alpha)$ ' derive ' $\exists \beta A(\beta)$ ', where $\beta$ is a variable) and Introduction of the Identity (derive $\alpha=\alpha$ ). Typical decompositional rules are Conjunction Elimination (from ' $A \wedge B$ ' derive ' $A$ '), Universal Instantiation (from ' $\forall \alpha A(\alpha)$ ' derive ' $A(\beta)$ '), Modus Ponens (from ' $A \supset B$ ' and ' $A$ ' derive ' $B$ ') and Modus Tollens (from ' $A \supset B$ ' and ' $\neg B$ ', derive $' \neg A$ ').

\footnotetext{
* I am indebted to the referees for providing useful comments on a former draft and for pointing my attention to some interesting related literature.

${ }^{\star \star}$ Peter Verdée is a post-doctoral fellow of the Fund for Scientific Research - Flanders.
} 
How can we interpret such a distinction? It is clarifying to look at this distinction from a information-theoretical point of view. We could consider rules as means to obtain more information from already derived information. Every step of a prove governed by rules reveals or discovers more information from the premises of the proof or from the laws of pure logic (in case of theorems) ${ }^{1}$. This process of revealing information goes in two directions. Decompositional rules reveal the information that is already fully present in the local premises. They make this information available for further proof steps. Compositional rules on the other hand somehow construct new information: they construct new (true) strings by bringing new symbols into the proof (in the case of Addition) or by combining already available strings and symbols into new more complex strings. The compositional steps of interesting proofs create new information about the premises, while the decompositional steps only analyze the premises. Compare it to a toy house built with Lego blocks. The decompositional steps correspond to the breaking down of the house into smaller parts, without doing anything new. The compositional steps correspond to building up the smaller parts into new creative houses or other constructions.

This distinction has an interesting computational aspect. One needs no goal directed procedure to apply all possible decompositional rules to a (finite) set of premises. This can be done in linear time, because the resulting formulas can only become smaller. Of course this is not the case for the compositional rules. One is, for example, able to apply the rule 'from $A$ derive $A \wedge A$ ' infinitely many times starting from one premise without ever obtaining the same formula. Hence, in order to have any chance at ever finding an interesting result by applying compositional rules, one needs a goal directed procedure, i.e. an algorithm that determines which rules to apply in order to be able to derive interesting formulas (goals).

My aim in this paper is threefold: first, I shall show that it is possible to devise a proof system for classical first order logic (henceforth called CL) in which one can properly distinguish the compositional from the decompositional rules. This might seem evident, but remember that fitch style proofs for example use conditional proofs. The status of the rule that allows one to derive an implication from a conditional proof is obviously a compositional rule. However, the conditional proof itself might also use decomposition, while the results of decomposition steps in conditional proofs definitely are not decompositions of the premises of the main proof. So, what would their status be? A usual axiom system has another problem: all steps, apart from Modus Ponens and the introduction of premises, are compositional (or decompositional, depending on

\footnotetext{
${ }^{1}$ According to the traditional view on information, logical deduction cannot provide new information. This position however is not anymore considered tenable by various contemporary scholars (cf. [7] and [11]). A more epistemic perspective on the matter, taking into account the fact that realistic agents do not have logical omniscience, calls for a less extreme position. Here I look at information from an intuitive epistemic stance. A more formal elaboration of my stance on information in deductive reasoning contexts is forthcoming.
} 
whether one sees axioms as available information). Obviously this makes the distinction rather useless. The proof system in this paper makes a clear distinction between compositional and decompositional steps. This distinction is even present at the object level: compositional steps result in formulas preceded by a special +-symbol. The logic $\mathbf{C}$, which I shall present here will formalize this proof system. The logic has a straight forward axiomatization and is therefore a usual Tarski logic: compact, monotonic, reflexive and transitive.

Secondly, I want to show that, using this proof system, one only needs one phase of decompositional steps followed by one phase of compositional steps in order to obtain the full consistent fragment of classical logic. This is implemented by requiring that, whenever 'new', composed formulas are derived, they are to be preceded by a special +-symbol and these +-preceded formulas are not to be decomposed. The decomposition phase, i.e. the phase in which formulas are only decomposed and no +-preceded formulas are obtained, could be interpreted as the analysis of the information present in the premises into the essential parts needed for the decomposition phase, where one is able to derive new, composed formulas. For the propositional level, the first phase more or less comes to analyzing the premises into a normal form. However, at the predicative level this is more complex.

Thirdly, and this is the main result, I want to show that one obtains a very nice paraconsistent logic called $\mathbf{C L}^{-}$by separating composition and decomposition. This first order logic is paraconsistent, in the sense that inconsistent premise sets do not lead to a trivial consequence set but the only thing one has to give up by moving to this special paraconsistent logic is transitivity (sometimes also called the 'Cut'-rule, i.e. if $\Gamma \vdash C$ for all $C \in \Delta$ and $\Gamma \cup \Delta \vdash B$ then $\Gamma \vdash B$ ). The logic is computationally not more complex than CL and has an elegant proof system. Moveover, and most importantly, everything useful that is derivable using CL, can also be derived using $\mathbf{C}$. This logic defines a consequence relation that is only weaker than the $\mathbf{C L}$-consequence relation in the following two aspects: (i) the trivial consequences of inconsistent premises are lost, but I suppose nobody will see this as a disadvantage and (ii) the consequence relation is no longer transitive. This last property might be considered as a disadvantage, but is solved by the fact that the logic $\mathbf{C}$ itself is transitive. Although $\mathbf{C}$ defines a weaker paraconsistent consequence relation than $\mathbf{C L}^{-}$in the usual $\mathbf{C L}$ language, the logics $\mathbf{C}$ and $\mathbf{C L}^{-}$have essentially the same proof theory and every reasoning process explicated by $\mathbf{C L}^{-}$can also be explicated by the logic $\mathbf{C}$.

The results in this paper are strongly inspired by important work by Batens and Provijn on so called goal directed or prospective proofs (cf. [1], [3], [4] and [12]). The proofs they present are essentially procedural and goal dependent, i.e. the correctness of a proof does not only depend on the premises of the proof but also on the goal of the proof (the formula that is to be derived). The propositional version of the logic $\mathbf{C L}^{-}$is an unexpected result of their goal directed proof system. It is my aim to (i) generalize their results on $\mathbf{C L}^{-}$to the first order level and (ii) to translate their proofs to usual non goal dependent, non procedural proofs. This has the important advantage that one can accumulate and reuse 
conclusions from a set of premises, i.e. old results obtained in proofs for some goal $A$ can be reused in proofs for a different goal $B$. This way I am able to reintroduce transitivity. Although I give up goal dependence, I shall show that my proofs can still very easily be turned into goal directed proofs, by adding some heuristic restrictions to the rules of the logic.

The logic presented in this paper is also related to Besnard and Hunter's quasi-classical logic (cf. [5], [9], and [10]) and Craig's Linear Reasoning (cf. [6]), which is inspired by Gentzen's famous cut-elimination theorem (cf. [8]). Both approaches separate compositional from decompositional steps in classical logic in a very similar way (apart from some details, the distinction is identical to the one I make). Craig, however, did not relate his results to paraconsistency, neither did he give a semantics for his proof system. Besnard and Hunter's fascinating QC logic is quite similar to the logic $\mathbf{C} \mathbf{L}^{-}$(apart from the fact that their consequence relation (in general) does not validate $\mathbf{C L}$-theorems), but lacks some of the attractive properties of the here presented logic $\mathbf{C}$, viz. (1) they do not present an axiomatization, (2) they do not introduce a symbol (like the +-symbol) to express the difference between decomposition and composition at object level, (3) their proofs do not show the attractive relevant logic interpretation that I shall explain in section 7, and (4) the semantic construction I shall present explicitly separates compositional and decomposition steps by means of gluts or gaps for all formulas, whereas their semantics is only an adequate semantics for the consequence relation $\mathbf{Q}$ (not for individual proof steps).

\section{$2 \quad$ Language and proof theory}

I start by presenting the formal languages that occur in this paper.

Let $\mathcal{L}$ be the language of $\mathbf{C L}$ with the logical symbols $\neg, \supset, \exists$, and $=$ (but without function symbols); $\mathcal{C}$ is the set of (letters for) individual constants, $\mathcal{V}$ the set of individual variables, $\mathcal{S}$ is the set of sentential letters, and $\mathcal{P}^{r}$ the set of predicates of rank $r \geq 0$-predicates of rank 0 will function as sentential letters. The members of $\mathcal{P}^{r}$ will be $P^{r}, Q^{r}, R^{r}, P_{1}^{r}, \ldots$. Let $\mathcal{F}$ denote the set of (possibly open) formulas of $\mathcal{L}$ and let $\mathcal{W}$ denote the set of closed formulas of $\mathcal{L}$. Let ${ }^{-} \mathcal{P}^{r}$ be the set of the members of $\mathcal{P}^{r}$ to which a $\neg$-symbol is added as a subscript, i.e. the set $\left\{P_{\neg}^{r}, Q_{\neg}^{r}, R_{\neg}^{r}, P_{1 \neg}^{r}, \ldots\right\}$ and let $\neg \mathcal{S}$ be the set of the members of $\mathcal{S}$ to which a $\neg$-symbol is added as a subscript, i.e. the set $\left\{p_{\neg}, q_{\neg}, r_{\neg}, p_{1 \neg}, \ldots\right\}$.

But the actual language of the logic $\mathbf{C}$ contains an extra + -symbol. Let $\mathcal{L}^{+}$ be exactly like $\mathcal{L}$, apart from the fact that the unary symbol + is added to the language. This symbol should only occur in front of formulas and should not be nested. $\mathcal{W}^{+}=\{+A \mid A \in \mathcal{W}\} \cup \mathcal{W} . \mathcal{F}^{+}$is defined analogously.

$\mathbb{P}$ denotes the set of all members of $\mathcal{W}$ in which no logical symbols (not even identity) occur and $\mathbb{I}=\{\alpha=\beta \mid \alpha, \beta \in \mathcal{C}\}$. $\mathbb{P}^{\urcorner}=\mathbb{P} \cup\{\neg A \mid A \in \mathbb{P}\}$ and $\mathbb{P}^{+}=\mathbb{P} \cup\{+A \mid A \in \mathbb{P}\} . \mathbb{I}^{+}$and $\mathbb{I}^{\urcorner}$are defined analogously.

Let us now turn to the description of the proof system. In order to facilitate this, I introduce the abbreviations $*$ and $\ddagger$ : where $A \in \mathcal{W}, * \neg A=A, * A=\neg A$ if $A$ is not of the form $\neg B, \ddagger+A=\mathrm{df} * A$, and $\ddagger A=\mathrm{df}+* A$. 
Lines of proofs do not contain just one formula, but a finite set of formulas between $\llbracket$ and $\rrbracket$. I use the sloppy notation $\llbracket A_{1}, \ldots, A_{n}, \Delta_{1}, \ldots, \Delta_{k}, B_{1}, \ldots, B_{m} \rrbracket$, where $\left\{A_{1}, \ldots, A_{n}\right\}$ and $\left\{B_{1}, \ldots, B_{n}\right\}$ are possibly empty, to denote the line $\llbracket\left\{A_{1}, \ldots, A_{n}, B_{1}, \ldots, B_{m}\right\} \cup \Delta_{1} \cup \ldots \cup \Delta_{k} \rrbracket$. The intuitive meaning of such lines is that at least one of the formulas in the set between $\llbracket$ and $\rrbracket$ on a derived line is derivable. But note that this interpretation is formally not entirely correct: the lines are actually a lot stronger than this, and behave like contraposable relevant conditionals, cf. section 7 .

I start by presenting an axiomatic proof system with 9 axiom schemata and 3 rules $^{2}$.

$$
\begin{aligned}
& \llbracket * A,+A \rrbracket \\
& \llbracket+* A,+A \rrbracket
\end{aligned}
$$$$
\llbracket A, B,+\neg(A \vee B) \rrbracket
$$

$$
\begin{aligned}
& \llbracket * B,+(A \vee B) \rrbracket \\
& \llbracket+\alpha=\alpha \rrbracket
\end{aligned}
$$

$$
\llbracket * A(\alpha),+\exists x A(x) \rrbracket
$$

$\llbracket+A(\alpha),+* A(\beta),+\neg \alpha=\beta \rrbracket$

$\llbracket+\neg \alpha=\beta,+\neg \beta=\gamma, \alpha=\gamma \rrbracket$

$\llbracket+\neg \alpha=\beta, \beta=\alpha \rrbracket$

TRANS from $\llbracket A, \Delta_{1} \rrbracket$ and $\llbracket \ddagger A, \Delta_{2} \rrbracket$ derive $\llbracket \Delta_{1} \cup \Delta_{2} \rrbracket$

$\mathrm{UG} \quad$ if $\beta \in \mathcal{C}$ does not occur in $\Delta \cup \Gamma \cup\{A(\alpha)\}$, from $\llbracket \Delta,+A(\beta) \rrbracket$ derive $\llbracket \Delta,+\neg \exists \alpha * A(\alpha) \rrbracket$

Definition 1. A C-proof from a set of premises $\Gamma$ is a list of lines of the form $\llbracket \Delta \rrbracket$ where $\Delta \subset \mathcal{W}^{+}$is finite and each line is an instance of one of the axiom schemata AS1-9 or the result of an application of one of the rules TRANS, PREM or $U G$.

Definition 2. Where $\Gamma \cup\{A\} \subset \mathcal{W}^{+}, \Gamma \vdash_{\mathbf{C}} A$ iff there is a $\mathbf{C}$-proof from premises $\Gamma$ in which $\llbracket A \rrbracket$ occurs on a line. Where $\Gamma \cup \Delta \subset \mathcal{W}^{+}, \Gamma \vdash_{\mathbf{C}} \llbracket \Delta \rrbracket$ iff there is a $\mathbf{C}$-proof from premises $\Gamma$ in which $\llbracket \Delta \rrbracket$ occurs on a line.

This completes the definition of the proof system C. I now introduce a more intuitive rule system called $\mathbf{C 2}$ (with many redundant, derivable rules). It inherits the 3 rules of $\mathbf{C}$, but 15 rules are added.

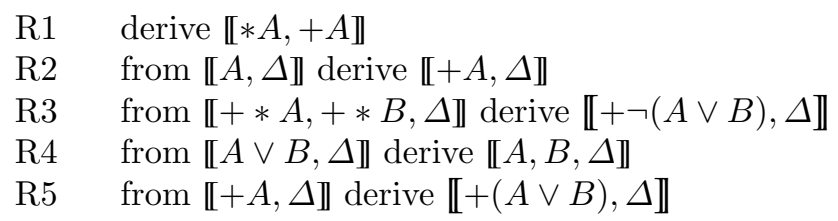

\footnotetext{
${ }^{2}$ Concerning the rule UG: if all $\beta \in \mathcal{C}$ already occur in $\Gamma$, one may introduce dummy constants in $\mathbf{C}$-proofs, but the formulas that are the conclusions of the proof should not contain these dummy constants
} 
R6 from $\llbracket+B, \Delta \rrbracket$ derive $\llbracket+(A \vee B), \Delta \rrbracket$

R7 from $\llbracket \neg(A \vee B), \Delta \rrbracket$ derive $\llbracket * A, \Delta \rrbracket$

R8 from $\llbracket \neg(A \vee B), \Delta \rrbracket$ derive $\llbracket * B, \Delta \rrbracket$

R9 derive $\llbracket+\alpha=\alpha \rrbracket$

R10 $\llbracket+\neg \alpha=\beta,+\neg \beta=\gamma, \alpha=\gamma \rrbracket$

$\mathrm{R} 11 \llbracket+\neg \alpha=\beta, \beta=\alpha \rrbracket$

R12 from $\llbracket+A(\alpha), \Delta \rrbracket$ derive $\llbracket+\exists x A(x), \Delta \rrbracket$

R13 from $\llbracket \neg \exists x A(x), \Delta \rrbracket$ derive $\llbracket * A(\alpha), \Delta \rrbracket$

R14 from $\llbracket A(\alpha), \Delta \rrbracket$ and $\llbracket * A(\beta), \Delta \rrbracket$ derive $\llbracket+\neg \alpha=\beta, \Delta \rrbracket$

R15 from $\llbracket \alpha=\beta, \Delta \rrbracket$ and $\llbracket A(\alpha), \Delta \rrbracket$ derive $\llbracket+A(\beta), \Delta \rrbracket$

Definition 3. A C2-proof from a set of premises $\Gamma$ is a list of lines of the form $\llbracket \Delta \rrbracket$ where $\Delta \subset \mathcal{W}^{+}$and each line is the result of an application of one of the rules TRANS, PREM or UG or R1-R15.

Definition 4. $\Gamma \vdash_{\mathbf{C} 2} A$ iff there is a $\mathbf{C}$-proof from premises $\Gamma$ in which $\llbracket A \rrbracket$ occurs on a line. $\Gamma \vdash_{\mathbf{C} 2} \llbracket \Delta \rrbracket$ iff there is a $\mathbf{C}$-proof from premises $\Gamma$ in which $\llbracket \Delta \rrbracket$ occurs on a line.

The proof systems $\mathbf{C}$ and $\mathbf{C 2}$ lead to exactly the same consequence relation. This is stated in the following theorem, which can easily be proved by showing that all $\mathbf{C}$-axioms can be derived using $\mathbf{C} 2$ rules and vice versa.

Theorem 1. $\Gamma \vdash_{\mathbf{C}} \llbracket \Delta \rrbracket$ iff $\Gamma \vdash_{\mathbf{C} 2} \llbracket \Delta \rrbracket$.

C2 has the advantage of yielding more natural proofs and explicitly showing the difference between composition and decomposition, while $\mathbf{C}$ is metatheoretically more elegant.

In both $\mathbf{C}$ and $\mathbf{C} \mathbf{2}$ one is able to define the other usual connectives in the usual way, i.e. $A \supset B=\mathrm{df}_{\mathrm{df}} * A \vee B, A \wedge B=\mathrm{df}_{\mathrm{df}} \neg(* A \vee * B), A \equiv B={ }_{\mathrm{df}}(A \supset$ $B) \wedge(B \supset A)$, and $\forall \alpha A(\alpha)={ }_{\mathrm{df}} \neg \exists \alpha * A(\alpha)$. In the example proofs, I shall use these defined symbols as abbreviations for their definientia.

Where $\mathbf{L}$ is a logic, $\mathcal{W}^{\prime}$ is the language of $\mathbf{L}$, and $\Gamma \subseteq \mathcal{W}^{\prime}$, let $C n_{\mathbf{L}}(\Gamma)=\mathrm{df}$ $\left\{A \in \mathcal{W}^{\prime} \mid \Gamma \vdash_{\mathbf{L}} A\right\}$. The following theorem will be proved later (in section 7 as theorem 9), but $\mathbf{C}$ is a logic that is derived from $\mathbf{C L}$ and the $\mathbf{C}$-proofs are meant to prove $\mathbf{C L}$-consequences, so this theorem is essential to understand the function of $\mathbf{C}$-proofs; it says that one can prove every $\mathbf{C L}$-consequence of every premise set $\Gamma \subset \mathcal{W}$ with a $\mathbf{C}$-proof, as far as $C n_{\mathbf{C L}}(\Gamma)$ is not trivial ${ }^{3}$.

Theorem 2. If there is some $C$ such that $\Gamma \nvdash_{\mathbf{C L}} C$, then $\Gamma \vdash_{\mathbf{C}}+A$ iff $\Gamma \vdash_{\mathbf{C L}} A$.

Although our proofs are strong enough to prove all useful CL-consequences, they respect the requirements for the behavior of the +-symbol: (i) every time a new, more complex formula is derived, it is preceded by a + -symbol (for example $\{P a\} \vdash_{\mathbf{C}}+\exists x P x, \vdash_{\mathbf{C}}+a=a,\{P a, P b\} \vdash_{\mathbf{C}}+(P a \wedge P b)$ and $\{P a \vee$

\footnotetext{
${ }^{3}$ Remark that this is not a weakness: if $C n_{\mathbf{C L}}(\Gamma)$ is trivial, proving $\mathbf{C L}$-consequences of $\Gamma$ is quite useless anyway.
} 
$P b\} \vdash_{\mathbf{C}}+(P b \vee P a)$ but $\{P a\} \nvdash_{\mathbf{C}} \exists x P x, \nvdash_{\mathbf{C}} a=a,\{P a, P b\} \nvdash_{\mathbf{C}} P a \wedge P b$ and $\left.\{P a \vee P b\} \nvdash_{\mathbf{C}} P b \vee P a\right)$ and on the other hand (ii) a formula which is preceded by a + -symbol is never decomposed $\left(\{+\forall x(P x \wedge R x)\} \nvdash_{\mathbf{C}}+P a\right.$ and $\left.\{+(P a \supset P b), P a\} \nvdash_{\mathbf{C}} P b\right)$. The only case where the requirements for the +symbol are slightly loosened is the case of identity. In order to obtain an elegant metatheory, I had to allow $\{a=b, b=c\} \vdash_{\mathbf{C}} a=c,\{a=b\} \vdash_{\mathbf{C}} b=a$ and $\{a=b\} \vdash_{\mathbf{C}} a=a$, although, strictly speaking, the ideas from section 1 would have required that these conclusions would only be allowed when preceded by a +-symbol, as e.g. $a=a$ or $b=a$ or their negations are not subformulas of $a=b$. Remark, however, that this is not a severe violation of the principles. Unlike in the case of e.g. what is sometimes called the stuttering rule $(A \vdash A \wedge A)$, which is not valid in $\mathbf{C}$, these identity transitivity and symmetry rules can never result in infinitely many + -free consequences of finitely many premises. For example the only +-free $\mathbf{C}$-consequences of $\{a=b, b=c\}$ are $\{a=a, a=b, a=c, b=a, b=$ $b, b=c, c=a, c=b, c=c\}$. As a consequence, these identity rules might also be seen as rules that result in a special kind of decomposition steps, rather than composition steps. Do not confuse this special case with normal identity cases, e.g. $\{P a, a=b\} \nvdash_{\mathbf{C}} P b$ and $\{P a\} \nvdash_{\mathbf{C}} a=a$, as the normal cases are perfectly in line with the requirements.

Finally, the attention of the reader should be pointed at the paraconsistency of $\mathbf{C}$, i.e. for every $A \in \mathcal{W}^{+}$, there is a $B \in \mathcal{W}^{+}$such that $A, \neg A \nvdash_{\mathbf{A}} B$. One can prove this by means of the paraconsistency of the semantics of $\mathbf{C}$ defined in Section 4 plus the soundness of the proof theory of $\mathbf{C}$ with respect to its semantics (cf. Section 5). However, to get a hint of how explosion is avoided, observe that there is no immediate Ex Falso Quodlibet rule in C. Also, the alternative way to derive explosion always involves composing a new formula which is later decomposed (for example by applying Addition and Disjunctive Syllogisme to an inconsistency: in case we have $p$ and $\neg p$, simply apply $p / p \vee q$ (Addition) and $\neg p, p \vee q / q$ (Disjunctive Syllogism) $-p \vee q$ as a newly composed formula which is decomposed later). Precisely this is blocked in $\mathbf{C}$ whence this blockage makes explosion impossible and the logic paraconsistent.

\section{$3 \quad$ Examples}

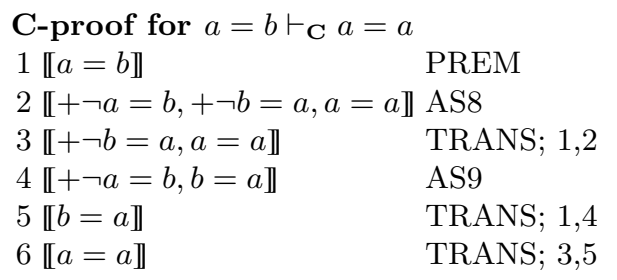




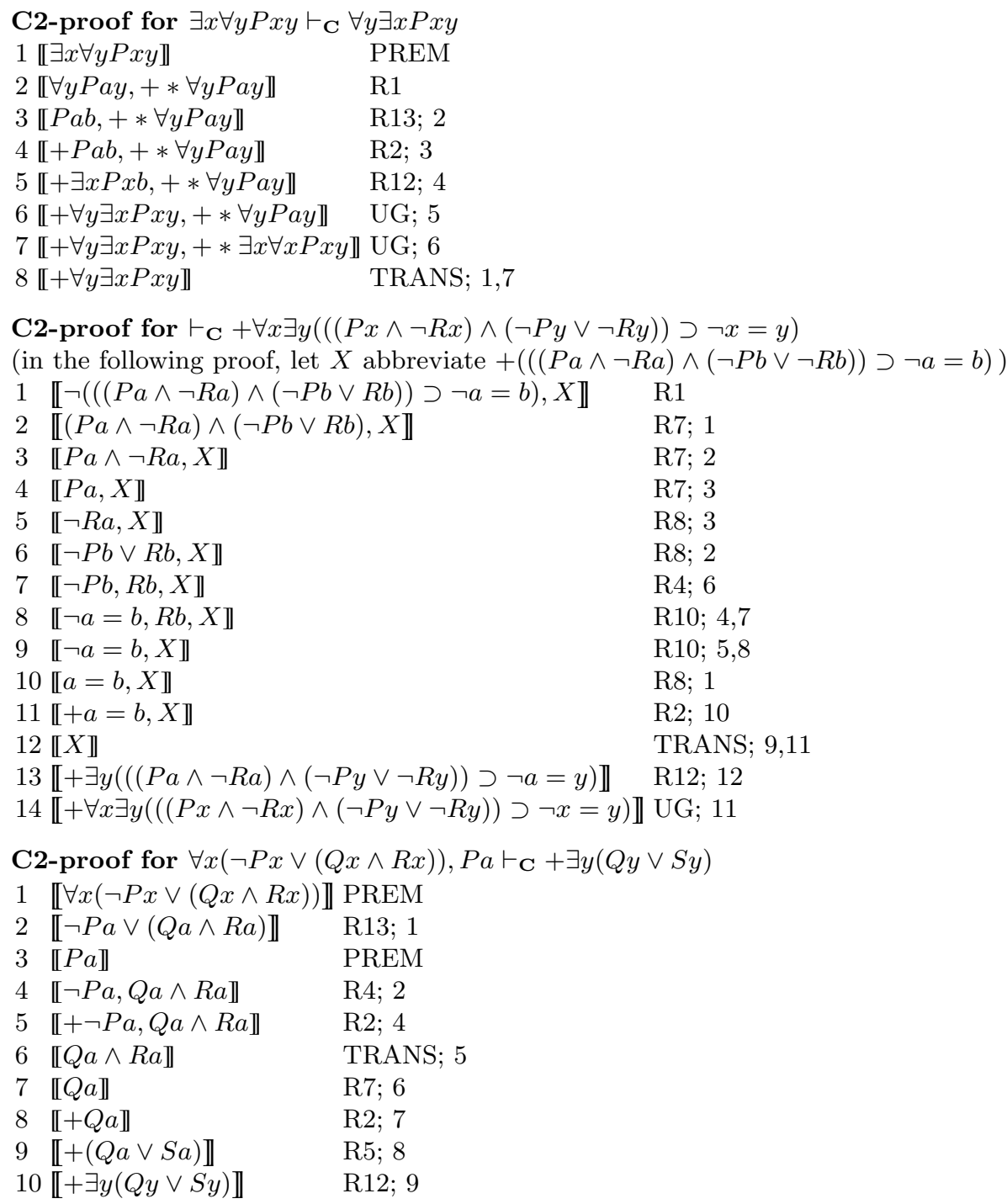

\section{Semantics}

Because this proof system (although it is a proof system for (consistent) CL) shows, thanks to its +-symbol and its paraconsistency, behavior that is quite different from $\mathbf{C L}$, it should not come as a surprise that one needs a completely different semantics for $\mathbf{C}$.

We shall use pseudo-constants to present the semantics as efficiently as possible. They do not occur in the actual object language (in premises, proofs or conclusions) but only in the semantics. The set $\mathcal{O}$ is the set of pseudo-constants. The language $\mathcal{L}_{o}$ is the language $\mathcal{L}$ enriched with these constants. The sets 
$\mathcal{F}_{o}, \mathcal{W}_{o}, \mathbb{P}_{o}, \mathbb{I}_{o}, \mathcal{F}_{o}^{+}, \mathcal{W}_{o}^{+}, \mathbb{P}_{o}^{+}, \mathbb{I}_{o}^{+}, \mathbb{P}_{o}^{\urcorner}$, and $\mathbb{I}_{o}$ are identical to the respective sets without subscript $o$, except for the replacement of $\mathcal{C}$ by $\mathcal{C} \cup \mathcal{O}$ in their definitions.

The semantics I am about to present is perfectly deterministic but has the unusual property that the assignment assigns (unrelated) truth values to all formulas (not only the primitive ones). This technical choice is made in order to ensure that all formulas without a +-symbol show a logical gap (the normal truth condition for the formula might be true, whilst the formula itself is false) and all formulas with a +-symbol show a logical glut (the normal truth condition for the formula might be false, whilst the formula itself is true). In this way we obtain that our requirements about +-symbols are satisfied.

A C-model $M$ is a triple $\langle v, w, D\rangle$, where $D$ (the domain) is some set of objects, $w: \mathcal{W}_{o}^{+} \rightarrow\{0,1\}$ is an assignment function which assigns a truth value to every formula, without respecting the structure of the formula, and $v$ is an assignment function that has the following properties:

(i) $\quad v: \mathcal{C} \cup \mathcal{O} \rightarrow D \quad($ with $D=\{v(\alpha) \mid \alpha \in \mathcal{C} \cup \mathcal{O}\})$

(ii) $v: \mathcal{S} \cup \neg \mathcal{S} \rightarrow\{0,1\}$

(iii) $v: \mathcal{P}^{r} \cup \mathcal{P}^{r} \rightarrow \wp\left(D^{(r)}\right) \quad$ (for every $r \geq 1$ ).

(iv) $v:\{\neg \cdot=\cdot, \cdot=\cdot,+\neg \cdot=\cdot\} \rightarrow \wp\left(D^{(2)}\right)$

Let $I_{v}(A)$, where $v$ is an assignment function with the properties mentioned above and $A$ is an atomic formula, be defined by ' $I_{v}\left(\pi^{r} \alpha_{1} \ldots \alpha_{r}\right)={ }_{d f}$ $\left\langle v\left(\alpha_{1}\right), \ldots, v\left(\alpha_{r}\right)\right\rangle \in v\left(\pi^{r}\right)^{\prime},{ }^{\prime} I_{v}\left(\neg \pi^{r} \alpha_{1} \ldots \alpha_{r}\right)=_{d f}\left\langle v\left(\alpha_{1}\right), \ldots, v\left(\alpha_{r}\right)\right\rangle \in v\left(\pi_{\neg}^{r}\right)^{\prime}$, ' $I_{v}(\sigma)=_{d f} v(\sigma)=1$ ', ' $I_{v}(\neg \sigma)=_{d f} v\left(\sigma_{\neg}\right)=1$ ', ' $I_{v}(\alpha=\beta)=\langle v(\alpha), v(\beta)\rangle \in v(\cdot=$ .)', ' $I_{v}(\neg \alpha=\beta)=\langle v(\alpha), v(\beta)\rangle \in v(\neg \cdot=\cdot)$ ', and ' $I_{v}(+\neg \alpha=\beta)=\langle v(\alpha), v(\beta)\rangle \in$ $v(+\neg \cdot=\cdot)$ '. Let $T_{M}(\beta)$, where $M$ is a model $\langle v, w, D\rangle$ and $\beta \in D$, abbreviate the semantic statement 'either $\langle\beta, \beta\rangle \in v(\neg \cdot=\cdot)$ or there is a $r$-ary predicate $\pi$ and $\alpha_{1}, \ldots, \alpha_{r-1} \in D$, such that $\left\langle\alpha_{1}, \ldots, \alpha_{i}, \beta, \alpha_{i+1}, \ldots, \alpha_{r-1}\right\rangle \in v\left(\pi^{r}\right) \cap v\left(\pi_{\neg}^{r}\right)$ '.

Definition 5. The function $\ddagger$ which maps metalinguistic semantic expressions to metalinguistic semantic expressions is recursively defined by means of the following clauses (between quotation marks) - note that I write square brackets to avoid confusion between function brackets and brackets in semantic expressions: " $\ddagger \mathrm{A}$ or $\mathrm{B}]=d f \ddagger \mathrm{A}$ and $\ddagger \mathrm{B} ", " \ddagger[\mathrm{A}$ and $\mathrm{B}]=d f \ddagger \mathrm{A}$ or $\ddagger \mathrm{B}$ ”, " $\ddagger$ for all $\beta \in$ $\mathcal{C} \cup \mathcal{O}, \mathrm{A}]=d f$ there exists a $\beta \in \mathcal{C} \cup \mathcal{O}$ such that $\ddagger \mathrm{A} ", " \ddagger[$ there exists a $\beta \in \mathcal{C} \cup \mathcal{O}$ such that $\mathrm{A}]={ }_{d f}$ for all $\beta \in \mathcal{C} \cup \mathcal{O}, \ddagger \mathrm{A} ", " \ddagger[w(A)=1]={ }_{d f} w(A)=0$ or $w(\ddagger A)=$ 1 ", “ $\left[I_{v}(A)\right]=d f$ not $I_{v}(A)$ or $I_{v}(\neg A)$ " where $A \in \mathbb{P}, " q\left[I_{v}(\alpha=\beta)\right]=d f$ not $I_{v}(\alpha=\beta)$ or $I_{v}(+\neg \alpha=\beta)$ ", " $\left[\right.$ not $I_{v}(A)$ or $\left.I_{v}(\neg A)\right]={ }_{d f} I_{v}(A)$ " where $A \in \mathbb{P}$, $"\left[v(\alpha) \neq v(\beta)\right.$ or $\left.T_{M}(v(\alpha))\right]=d f v(\alpha)=v(\beta) ", " 4[v(\alpha)=v(\beta)]=d f v(\alpha) \neq$ $v(\beta)$ or $T_{M}(v(\alpha))$ " and finally $"\left[v_{M}(A)=i\right]=d f v_{M}(\ddagger A)=i$ ", where $i \in\{0,1\}$.

The valuation function $v_{M}: \mathcal{W}_{o}^{+} \rightarrow\{0,1\}$, where $M=\langle v, w, D\rangle$ is a C-model, is defined by the following clauses.

S1 $\quad$ where $A \in \mathbb{P}, v_{M}(A)=1$ iff $w(A)=1$ and $I_{v}(A)$ 
where $A \in \mathbb{P}, v_{M}(\neg A)=1$ iff $w(\neg A)=1$ and (not $I_{v}(A)$ or $I_{v}(\neg A)$ )

$v_{M}(\neg \neg A)=1$ iff $w(\neg \neg A)=1$ and $v_{M}(A)=1$

$v_{M}(\neg(A \vee B))=1$ iff $w(\neg(A \vee B))=1$ and $v_{M}(* A)=1$ and $v_{M}(* B)=1$

$v_{M}(A \vee B)=1$ iff $w(A \vee B)=1$ and $\left(v_{M}(+* A)=0\right.$ or $\left.v_{M}(B)=1\right)$ and $\left(v_{M}(+* B)=0\right.$ or $\left.v_{M}(A)=1\right)$

$v_{M}(\neg \exists \alpha A(\alpha))=1$ iff $w(\neg \exists \alpha A(\alpha))=1$ and for all $\beta \in \mathcal{C} \cup \mathcal{O}, v_{M}(\neg A(\beta))=$ 1

$v_{M}(\exists \alpha A(\alpha))=1$ iff $w(\exists \alpha A(\alpha))=1$ and there exists a $\beta \in \mathcal{C} \cup \mathcal{O}$, such that $v_{M}(A(\beta))=1$

$v_{M}(\neg \alpha=\beta)=1$ iff $w(\neg \alpha=\beta)=1$ and $\left(v(\alpha) \neq v(\beta)\right.$ or $\left.T_{M}(v(\alpha))\right)$

$v_{M}(\alpha=\beta)=1$ iff $\left(I_{v}(\alpha=\beta)\right.$ or $\left.w(\beta=\beta)=1\right)$ and $v(\alpha)=v(\beta)$

$v_{M}(+A)=1$ iff $\ddagger\left[v_{M}(* A)=1\right]$

Clauses S2, S8 and S10 deserve a little extra attention. Clause S2 and S8 introduce another type of gluts than the ones introduced to fulfil the requirement for the +-symbol. This glut is a typical negation glut and ensures the paraconsistency of the logic. Remark that the logic needs to be paraconsistent as we obviously do not want to decompose formulas $A$ and $\neg A$ into an arbitrary formula $B$. Clause S10 abbreviates all clauses for formulas of the form $+A$. They are generated by $\ddagger$-transforming the clause for $* A$ in line with Definition 5 .

Given these criteria for being a $\mathbf{C}$ valuation function $v_{M}$ we can obtain the following properties (for every $A \in \mathcal{W}_{o}^{+}$and every C-model $M$ ) by a straight forward mathematical induction on the complexity of $A$.

Lemma 1. 1. if $v_{M}(A)=1$ then $v_{M}(+A)=1$

2. $v_{M}(A)=1$ or $v_{M}(\ddagger A)=1$

Finally we have all the means to determine when a $\mathbf{C}$-model satisfies a formula and to define semantic consequence.

Definition 6. Satisfaction.

Where $A \in \mathcal{W}^{+}$and $M$ is a $\mathbf{C}$-model, $M \models A$ iff $v_{M}(A)=1$.

Definition 7. Semantic C-consequence.

Where $\Gamma \cup\{A\} \subset \mathcal{W}^{+}, \Gamma \vDash_{\mathbf{C}} A$ iff $M \models A$ for every model $M$ such that $M \models B$ for all $B \in \Gamma$.

\section{Soundness, completeness and other important properties}

We start by some important properties of the logic $\mathbf{C}$. The next interesting Lemma might be seen as an alternative for the usual deduction theorem. It is proven in the Appendix of this paper.

Lemma 2. If $\Delta^{\prime} \subset \Delta$ and $\Gamma \cup\left\{\ddagger B \mid B \in \Delta^{\prime}\right\} \vdash_{\mathbf{C}} \llbracket \Delta-\Delta^{\prime} \rrbracket$, then there is a $\Theta \subset\left\{\ddagger B \mid B \in \Delta^{\prime}\right\}$ such that $\Gamma \cup \Theta \vdash_{\mathbf{C}} \llbracket \Delta-\Delta^{\prime} \rrbracket$ or $\Gamma \vdash_{\mathbf{C}} \llbracket \Delta \rrbracket$

This lemma has some interesting corollaries. 
Corollary 1. If $\Gamma \cup A \vdash_{\mathbf{C}} B$ then $\Gamma \vdash_{\mathbf{C}} B$ or $\Gamma \vdash_{\mathbf{C}} \llbracket \ddagger A, B \rrbracket$.

Corollary 2. If $\Gamma \cup A \vdash_{\mathbf{C}} B$ then $\Gamma \vdash_{\mathbf{C}} B$ or $\Gamma \cup\{\ddagger B\} \vdash_{\mathbf{C}} \ddagger A$.

Corollary 3. If $\Gamma \cup\{A\} \vdash_{\mathbf{C}} B$ and $\Gamma \cup \Delta \cup\{\ddagger A\} \vdash_{\mathbf{C}} B$, then $\Gamma \cup \Delta \vdash_{\mathbf{C}} B$.

The next lemma immediately entails the soundness theorem for $\mathbf{C}$.

Lemma 3. If $\Gamma \vdash_{\mathbf{C}} \llbracket \Delta \rrbracket$ then for all $A \in \Delta, \Gamma \cup\{\ddagger B \mid B \in \Delta-\{A\}\} \vDash_{\mathbf{C}} A$.

Proving Lemma 3 is just a matter of letting every line of every proof correspond to its appropriate semantic $\mathbf{C}$-consequence and proving that every $\mathbf{C}$ rule corresponds to a correct fact about semantic $\mathbf{C}$-consequences. This is quite straight forward except maybe for the fact that one should be aware of the fact that $\llbracket A, A \rrbracket$ is identical to $\llbracket A \rrbracket$. The semantic fact that correspond to this is that $\Gamma \cup\{\ddagger A\} \vDash_{\mathbf{C}} A$ iff $\Gamma \vDash_{\mathbf{C}} A$, which is warranted by Lemma 1 .

Theorem 3. Soundness of C.

For every $\Gamma \cup\{A\} \subseteq \mathcal{W}^{+}$, if $\Gamma \vdash_{\mathbf{C}} A$ then $\Gamma \vDash_{\mathbf{C}} A$

The following completeness theorem is proved in the appendix of this paper.

Theorem 4. Completeness of $\mathbf{C}$.

For every $\Gamma \cup\{A\} \subseteq \mathcal{W}^{+}$, if $\Gamma \vDash_{\mathbf{C}} A$ then $\Gamma \vdash_{\mathbf{C}} A$

Other important but easily provable properties of $\mathbf{C}$ are listed in the following theorem.

Theorem 5. C is transitive, monotonic and reflexive. $\mathbf{C}$-proofs are recursive whence $\mathrm{Cn}_{\mathbf{C}}$ is semi-recursive.

Finally, observe that $\mathbf{C}$ is paraconsistent, in view of the fact that every premise set has a $\mathbf{C}$-model; the model that makes all primitive formulas that occur in the premises or of which the negation occurs in the premises true and all other primitive formulas false. This model will satisfy the premises and falsify at least one formula (on the condition that not all primitive formulas or their negations occur in the premises - this condition is always true for premise sets $\{A, \neg A\})$.

Theorem 6. C is paraconsistent, i.e. for every $A \in \mathcal{W}$, there is a $B \in \mathcal{W}^{+}$ such that $A, \neg A \nvdash_{\mathbf{C}} B$ and also $+A,+\neg A \nvdash_{\mathbf{C}} B$.

\section{A strong paraconsistent version of CL}

We now return to the relation between $\mathbf{C}$ and $\mathbf{C L}$. We start by defining an extension of consistent CL (this is the logic that allows all the normal CLconsequences of consistent premises and does not give any consequence for inconsistent premise sets). 
Definition 8. The logic $\mathbf{C L}^{-}$.

$\mathbf{C L}^{-}$uses the language $\mathcal{L}$ and where $\Gamma \cup\{A\} \in \mathcal{W}, \Gamma \vdash_{\mathbf{C L}^{-}} A$ iff $\Gamma \vdash_{\mathbf{C}}+A$

The propositional fragment of this logic is neatly characterized in [1] as the logic $\mathbf{Q}$. Proving that the propositional fragment of $\mathbf{C} \mathbf{L}^{-}$is indeed equivalent to this logic $\mathbf{Q}$ is quite straight forward but rather tedious.

I list the most important properties of $\mathbf{C L}^{-}$(most of them are easily provable, except maybe the last one - for this property observe that $A, \neg A,(A \vee B) \vee \neg(A \vee$ $B) \vdash_{\mathbf{C}} B$, which is provable by means of a proof of which the essential lines are $\llbracket A \vee B, \neg(A \vee B) \rrbracket, \llbracket A, B, \neg(A \vee B) \rrbracket$, and $\llbracket A, B, \neg A \rrbracket)$.

Theorem 7. 1. $\mathbf{C L}^{-}$is paraconsistent.

2. $\mathbf{C L}^{-}$is not transitive.

3. $\mathbf{C L}^{-}$is monotonic and reflexive.

4. $\mathbf{C L}^{-}$-proofs are recursive whence $\vdash_{\mathbf{C L}^{-}}$is a semi-recursive relation (if the set of premises is recursive).

5. $C n_{\mathbf{C L}^{-}}(\Gamma \cup\{\neg A \vee A \mid A \in \mathcal{W}\})=C n_{\mathbf{C L}}(\Gamma)$.

The semi-recursiveness of $\vdash_{\mathbf{C L}^{-}}$is quite important and remarkable. As far as my knowledge goes, there are no other existing paraconsistent logics that allow all the $\mathbf{C L}$-consequences of consistent premise sets AND have a positive test whenever the set of premises is recursive. Most paraconsistent logics are weaker than consistent CL (they invalidate $\mathbf{C L}$-rules like Disjunctive Syllogism or Addition). Those that are not weaker than consistent $\mathbf{C L}$ are usually non-monotonic (e.g. inconsistency adaptive logics, see [2], and consistent CL itself), which means that some consequences are revoked when the premise set is enriched with inconsistencies. Of course, this requires a full overview over the premises before one can be certain that some formula is a consequence. Consequently, these logics do not have a positive test (for infinite premise sets) whence they are not semi-recursive.

In what follows (and in the proofs in the appendix) let $\exists A$, where $A \in \mathcal{F}$, denote the existential closure of $A$ and let $\Gamma \vDash_{\mathbf{L}} A \sqcup B$ denote that $M \models A$ or $M \models B$, for all $\mathbf{L}$-models $M$ such that $M \models \Gamma$

We now jump to the adequateness of $\mathbf{C L}^{-}$(and hence also of $\mathbf{C}$ ) with respect to $\mathbf{C L}$. Proving soundness is straight forward: if every line $\llbracket \Delta \rrbracket$ is transformed into $\bigvee \Delta^{\prime}$, where $\Delta^{\prime}$ is $\Delta$ without +-symbols, every transformation is evidently a correct $\mathbf{C L}$-consequence of the premises without + -symbols.

Theorem 8. If $\Gamma \vdash_{\mathbf{C L}^{-}} A$ then $\Gamma \vdash_{\mathbf{C L}} A$

Completeness however is less straight forward. We first need two lemma's. The first lemma is easily provable in view of Theorem 8 and the fact that $A, \neg A \vdash_{\mathrm{CL}} B$.

Lemma 4. If $\Gamma \vdash_{\mathbf{C}}+\exists(D \wedge \neg D)$ for some $D \in \mathcal{F}$, then $C n_{\mathbf{C L}}(\Gamma)=\mathcal{W}$.

Lemma 5. If $\Gamma \vDash_{\mathbf{C}}+A \sqcup+B \sqcup+C$, then $\Gamma \vDash_{\mathbf{C}}+A, \Gamma \vDash_{\mathbf{C}}+B, \Gamma \vDash_{\mathbf{C}}+C$ or $\Gamma,+* A,+* B \vDash_{\mathbf{C}} C$. 
Now we finally have all the means to prove the (consistent premises) completeness of $\mathbf{C}$ and $\mathbf{C L}^{-}$with respect to $\mathbf{C L}$. Remark that this theorem is exactly the same as Theorem 2. The proof of this theorem is in the appendix.

Theorem 9. If there is some $C$ such that $\Gamma \nvdash_{\mathbf{C L}} C$, then $\Gamma \vdash_{\mathbf{C L}^{-}} A$ iff $\Gamma \vdash_{\mathbf{C L}}$ A.

\section{A relevant logic interpretation for the lines of C-proofs}

The lines of $\mathbf{C}$-proofs are easily interpretable as suggested by the soundness theorem: a line $\llbracket \Delta \rrbracket$ in a proof from premises $\Gamma$ is interpreted as the expression "for all $A \in \Delta, \Gamma \cup\{\ddagger B \mid B \in \Delta-\{A\}\} \vdash_{\mathbf{C}} A$ ". This interpretation holds for all lines, but the rules of the proofs are not complete with respect to this interpretation, i.e. it is not the case that "if for every $A \in \Delta$ holds $\Gamma \cup\{\ddagger B \mid B \in \Delta-\{A\}\} \vdash_{\mathbf{C}} A$, then $\Gamma \vdash \llbracket \Delta \rrbracket$. A simple counterexample for this is the fact that $A \vee B, C \vee D \nvdash_{\mathbf{C}} \llbracket A, B, C, D \rrbracket$, while obviously $\{A \vee B, C \vee D,+\neg A,+\neg B,+\neg C\} \vdash_{\mathbf{C}} D,\{A \vee B, C \vee D,+\neg A,+\neg B,+\neg D\} \vdash_{\mathbf{C}} C$, $\{A \vee B, C \vee D,+\neg A,+\neg C,+\neg D\} \vdash_{\mathbf{C}} B$, and $\{A \vee B, C \vee D,+\neg B,+\neg C,+\neg D\} \vdash_{\mathbf{C}}$ $A$.

Actually, these lines of $\mathbf{C}$-proof are in fact relevant contraposable implications in disguise, where a line $\llbracket A, B, C, D \rrbracket$, for example, would, in a usual relevant logic like the logic $\mathbf{R}$, be written as $* A \rightarrow(* B \rightarrow(* C \rightarrow D))$. The reason why the simple interpretation explained above is not adequate is precisely this relevant character of the lines. In particular, the problem is related to the fact that our proofs do not allow for weakening. This property/rule is usually expressed as $A \rightarrow(B \rightarrow A)$ and is not valid in relevant logics (if $A$ is true anyhow, (the truth of) $B$ is irrelevant for the truth of $A$ ). In the case of $\mathbf{C}$-proofs weakening comes to deriving $\llbracket C, D \rrbracket$ from $\llbracket C \rrbracket$, which is evidently impossible. Another relevantly invalid property is $A \rightarrow(B \rightarrow(\neg A \rightarrow B))$. For our lines this comes to deriving $\llbracket A, B \rrbracket$ from lines $\llbracket A \rrbracket$ and $\llbracket B \rrbracket$, which is also not allowed. On the other hand all relevantly unproblematic consequences are allowed in $\mathbf{C}$-proofs (modus ponens, transitivity, contraction, contraposition).

The following interpretation for lines of $\mathbf{C}$-proofs is adequate with respect to C-proofs.

Definition 9. Where $\Gamma, \Delta \subset \mathcal{W}^{+}, \Gamma \vDash_{\mathbf{C}} \llbracket \Delta \rrbracket$ iff $\langle\Gamma, \Delta\rangle \in X$, where $X$ is the smallest set that satisfies the two following criteria

1. $\langle\Gamma,\{A\}\rangle \in X$ if $\Gamma \vDash_{\mathbf{C}} A$,

2. $\langle\Gamma \cup\{A\}, \Delta\rangle \in X$ if $\langle\Gamma, \Delta\rangle \in X$,

3. $\left\langle\Gamma \cup \Gamma^{\prime}, \Delta\right\rangle$ if $\Gamma \vDash_{\mathbf{C}} A$ and $\left\langle\Gamma^{\prime} \cup\{A\}\right.$, $\left.\llbracket \Delta \rrbracket\right\rangle$, and

4. $\langle\Gamma, \Delta\rangle$ if, for every $\Delta^{\prime} \subset \Delta,\left\langle\Gamma \cup\left\{\ddagger B \mid B \in \Delta^{\prime}\right\}, \Delta-\Delta^{\prime}\right\rangle \in X$ and, for every $\Theta \subset\left\{\ddagger B \mid B \in \Delta^{\prime}\right\},\left\langle\Gamma \cup \Theta, \Delta-\Delta^{\prime}\right\rangle \notin X$.

Theorem 10. For every $\Gamma \cup \Delta \subseteq \mathcal{W}^{+}, \Gamma \vdash_{\mathbf{C}} \llbracket \Delta \rrbracket$ iff $\Gamma \vDash_{\mathbf{C}} \llbracket \Delta \rrbracket$. 
Although this interpretation is not deterministic, it is quite interesting as it seems to be an elegant means to define the semantics of quite a rich relevant implication without reference to worlds and accessibility relations.

I shall now make the relation between $\mathbf{C}$ and relevant conditionals precise. Let $\mathcal{W}^{\rightarrow}$ be the set of formulas defined by $\mathcal{W} \subset \mathcal{W}^{\rightarrow}$ and $A \rightarrow B \in \mathcal{W}^{\rightarrow}$ iff $A, B \in \mathcal{W}^{\rightarrow}$. We need to define a translation function $\operatorname{tr}$ before we can proceed to defining relevant theoremhood based on the lines of $\mathbf{C}$-proofs.

1. where $n>0, B \in \mathcal{W}$ and $A_{1}, \ldots A_{n} \in \mathcal{W}^{\rightarrow}$,

$$
\begin{aligned}
& \operatorname{tr}\left(A_{1} \rightarrow\left(A_{2} \rightarrow\left(A_{3} \rightarrow \ldots\left(A_{n} \rightarrow B\right) \ldots\right)\right)\right)= \\
& \quad \llbracket \operatorname{tr}^{\prime}\left(A_{1}\right), \neq \operatorname{tr}^{\prime}\left(A_{2}\right), \neq \operatorname{tr}^{\prime}\left(A_{3}\right), \ldots, \ddagger \operatorname{tr}^{\prime}\left(A_{n}\right),+B \rrbracket
\end{aligned}
$$

2. where $A \in \mathcal{W}, \operatorname{tr}(A)=+A$ and $\operatorname{tr}^{\prime}(A)=A$

3. where $A_{1}, A_{2} \in \mathcal{W}^{\rightarrow} \operatorname{tr}^{\prime}\left(A_{1} \rightarrow A_{2}\right)=\neg \operatorname{tr}^{\prime}\left(A_{1}\right) \vee \operatorname{tr}^{\prime}\left(A_{2}\right)$

Definition 10. Let the logic $\vdash_{\mathbf{C r}}$ be defined by:

- the set of formulas of $\mathbf{C r}$ is $\mathcal{W}^{\rightarrow}$,

- where $A \in \mathcal{W}^{\rightarrow}, \vdash_{\mathbf{C r}} A$ iff $\vdash_{\mathbf{C}} \operatorname{tr}(A)$, and

- only theoremhood of $\mathbf{C r}$ is defined, no consequence relation.

It is easy to check that: (in the right column the corresponding translation to a $\mathbf{C}$-theorem is mentioned)

$$
\begin{array}{ll}
\nvdash_{\mathbf{C r}} B \rightarrow(A \rightarrow B) & \nvdash_{\mathbf{C}} \llbracket+* B,+* A,+B \rrbracket \\
\nvdash_{\mathbf{C r}} A \rightarrow(B \rightarrow B) & \nvdash_{\mathbf{C}} \llbracket+* A,+* B,+B \rrbracket \\
\nvdash_{\mathbf{C r}} A \rightarrow(\neg B \vee B) & \nvdash_{\mathbf{C}} \llbracket+* A,+(\neg B \vee B) \rrbracket \\
\nvdash_{\mathbf{C r}} B \rightarrow(\neg B \rightarrow A) & \nvdash_{\mathbf{C}} \llbracket+* B,+B,+A \rrbracket \\
\nvdash_{\mathbf{C r}} B \rightarrow(A \rightarrow(\neg B \rightarrow A) & \nvdash_{\mathbf{C}} \llbracket+* B,+* A,+B,+A \rrbracket \\
\vdash_{\mathbf{C r}} A \rightarrow A & \vdash_{\mathbf{C}} \llbracket+* A,+A \rrbracket \\
\vdash_{\mathbf{C r}} A \rightarrow(A \vee B) & \vdash_{\mathbf{C}} \llbracket+* A,+(A \vee B) \rrbracket \\
\vdash_{\mathbf{C r}}(A \wedge B) \rightarrow A & \vdash_{\mathbf{C}} \llbracket+*(A \wedge B),+A \rrbracket \\
\vdash_{\mathbf{C r}} A \rightarrow(B \rightarrow(A \wedge B)) & \vdash_{\mathbf{C}} \llbracket+* A,+* B,+(A \wedge B) \rrbracket \\
\text { where } A \in \mathcal{W} \text { is a CL-theorem, } \vdash_{\mathbf{C r}} A & \vdash_{\mathbf{C} \llbracket+A \rrbracket} \\
\vdash_{\mathbf{C r}}\left(A \rightarrow B \rightarrow((B \rightarrow C) \rightarrow(A \rightarrow C)) \vdash_{\mathbf{C}} \llbracket+*(* A \vee B),+*(* B \vee C),+* A,+C \rrbracket\right. \\
\vdash_{\mathbf{C r}}(A \rightarrow(B \rightarrow C)) \rightarrow(B \rightarrow(A \rightarrow C)) \vdash_{\mathbf{C}} \llbracket+*(* A \vee(* B \vee C)),+* B,+* A,+C \rrbracket
\end{array}
$$

It is still unclear whether $\mathbf{C r}$ is a fully relevant logic, but the above properties are quite promising. All straight forward irrelevances of CL (paradoxes of material implication) are eliminated for the $\rightarrow$-implication, while at the same time it is crystal clear that $\rightarrow$ is a fairly strong conditional (all axioms of the standard relevant logic $\mathbf{R}$ are valid). Although it seems likely that $\mathbf{C r}$ is a useful relevant logic, it has an elegant proof theory which has an adequate semantics that does not refer to worlds or accessibility relations. This is a rather remarkable result.

Remark that this conditional has some unusual properties. For example, Disjunctive Syllogism is valid (i.e. $\vdash_{\mathbf{C r}}(A \vee B) \rightarrow(\neg A \rightarrow B)$ ), and so are Addition (i.e. $\left.\vdash_{\mathbf{C r}} A \rightarrow(A \vee B)\right)$, Transitivity (i.e. $\vdash_{\mathbf{C r}}(A \rightarrow B) \rightarrow((B \rightarrow C) \rightarrow(A \rightarrow$ $C))$ ), and Modus Ponens (i.e. $\left.\vdash_{\mathbf{C r}} A \rightarrow((A \rightarrow B) \rightarrow B)\right)$. From the validity of these rules, one would expect that Ex Falso Quodlibet (i.e. $A \rightarrow(\neg A \rightarrow B)$ ) is 
also validated in $\mathbf{C r}$, but this is NOT the case. This is possible due to the fact that the metatheoretic version of Modus Ponens (from $\vdash A$ and $\vdash A \rightarrow B$, derive $\vdash B)$ is NOT valid in $\mathbf{C r}$.

\section{Procedures for proof generation}

In the first section, I mentioned that the ideas for the logic $\mathbf{C}$ are inspired by the goal directed proof system defined by Batens and Provijn. The main objective of those goal directed proofs is to push the heuristics a reasoner uses to construct a proof for a given formula into the proof itself. The goal directed proofs do not allow useless (but essentially correct) proof steps. For example, if one wants to derive $q$ from $p$ and $p \supset q$, deriving $p \vee r$ will (although evidently correct in CL) never be considered as a wise step in a proof. Goal directed proofs give a formal criterion to distinguish between potentially useful and useless steps in a proof with a certain goal. Evidently, these goal directed proofs, in which one avoids useless steps, can easily be turned into algorithms that construct proofs. Hence, they also form (partial) decision methods for logical consequence.

My aim was precisely to eliminate the goal dependency from the goal directed proofs (and thereby making transitive reasoning and a usual semantics and axiomatization for $\mathbf{C L}^{-}$possible). So I had to lose part of the advantage and the original aim of the goal directed proofs. However, the goal directed element can easily be added on top of the C1-proofs. Making C1-proofs as elegant and insightful as the original goal directed proofs is just a matter of adding some minor heuristic information to the proofs; the logical rules do not need to change. Such an enterprise, however, is not substantial for my present purpose. 


\section{Bibliography}

[1] Diderik Batens. It might have been classical logic. Logique et Analyse. URL http://logica.ugent.be/centrum/preprints/PCLs.pdf.

[2] Diderik Batens. Inconsistency-adaptive logics. In Ewa Orłowska, editor, Logic at Work. Essays Dedicated to the Memory of Helena Rasiowa, pages 445-472. Physica Verlag (Springer), Heidelberg, New York, 1999.

[3] Diderik Batens. A formal approach to problem solving. In Claudio Delrieux and Javier Legris, editors, Computer Modeling of Scientific Reasoning, pages 15-26. Universidad Nacional del Sur, Bahia Blanca, Argentina, 2003.

[4] Diderik Batens and Dagmar Provijn. Pushing the search paths in the proofs. A study in proof heuristics. Logique et Analyse, 173-175:113-134, 2001. Appeared 2003.

[5] Philippe Besnard and Anthony Hunter. Quasi-classical logic: Nontrivializable classical reasoning from incosistent information. In Proceedings of the European Conference on Symbolic and Quantitative Approaches to Reasoning and Uncertainty, pages 44-51, London, UK, 1995. SpringerVerlag.

[6] William Craig. Linear reasoning. a new form of the herbrand-gentzen theorem. The Journal of Symbolic Logic, 22(3):250-268, 1957.

[7] Marcello D'Agostino and Luciano Floridi. The enduring scandal of deduction. Synthese, 167(2), 2009.

[8] Gerhard Gentzen. Untersuchungen ber das logische schlieen. ii. Mathematische Zeitschrift, 39:405-431, 1935. ISSN 0025-5874.

[9] Anthony Hunter. Reasoning with contradictory information using quasiclassical logic. Journal of Logic and Computation, 10:677-703, 1999.

[10] Anthony Hunter. A semantic tableau version of first-order quasi-classical logic. In Salem Benferhat and Philippe Besnard, editors, Symbolic and Quantitative Approaches to Reasoning with Uncertainty, volume 2143 of Lecture Notes in Computer Science, pages 544-555. Springer Berlin / Heidelberg, 2001.

[11] Mark Jago. Logical information and epistemic space. Synthese, 167(2), 2009.

[12] Dagmar Provijn. Prospectieve dynamiek. Filosofische en technische onderbouwing van doelgerichte bewijzen en bewijsheuristieken. $\mathrm{PhD}$ thesis, Universiteit Gent (Belgium), 2005. Unpublished PhD thesis. 


\section{Appendix: metaproofs}

\section{Sketch of the proof of Lemma 2}

Suppose $\Delta^{\prime} \subset \Delta, \Gamma \cup\left\{\ddagger B \mid B \in \Delta^{\prime}\right\} \vdash_{\mathbf{C}} \llbracket \Delta-\Delta^{\prime} \rrbracket$ and for all $\Theta \subset\left\{\ddagger B \mid B \in \Delta^{\prime}\right\}$, $\Gamma \cup \Theta \nvdash_{\mathbf{C}} \llbracket \Delta-\Delta^{\prime} \rrbracket$. Hence there is a proof in which $\llbracket \Delta-\Delta^{\prime} \rrbracket$ is derived and every $\llbracket \ddagger B \rrbracket$, where $B \in \Delta^{\prime}$, is used as a local premise in the derivation tree of $\llbracket \Delta-\Delta^{\prime} \rrbracket$. Now it is possible to replace every such $\llbracket \ddagger B \rrbracket$ by $\llbracket \ddagger B, B \rrbracket$ (remember that this is an axiom). The rest of the proof can remain the same, apart from the fact that for every line which is in the derivation tree on a branch that contains a line $\llbracket \ddagger B \rrbracket$, where $B \in \Delta^{\prime}, B$ should be added as an element of that line. The result will be that the line $\llbracket \Delta-\Delta^{\prime} \rrbracket$ is transformed into $\llbracket \Delta \rrbracket$ whence the proof for $\Gamma \cup\left\{\ddagger B \mid B \in \Delta^{\prime}\right\} \vdash_{\mathbf{C}} \llbracket \Delta-\Delta^{\prime} \rrbracket$ is transformed into a proof for $\Gamma \vdash_{\mathbf{C}} \llbracket \Delta \rrbracket$.

\section{Sketch of the proof of Theorem 4}

Let $\mathcal{L}_{p}$ be exactly as $\mathcal{L}_{o}$ except for the replacement of $\mathcal{O}$ by $\mathcal{O}^{\prime}$, which is an arbitrary, countably infinite subset of $\mathcal{O} . \mathcal{W}_{p}, \mathbb{P}_{p}, \mathcal{L}_{p}^{+}, \mathcal{W}_{p}^{+}$, and $\mathbb{P}_{p}^{+}$are defined analogously. Let $\mathcal{D}_{p}={ }_{\mathrm{df}}\left\{\llbracket \Delta \rrbracket \mid \Delta \subset \mathcal{W}_{p}^{+}\right\}$.

Let a $\mathbf{C} *$-proof from $\Gamma \in \mathcal{W}_{p}^{+} \cup \mathcal{D}_{p}$ be a list of lines such that every line is either a line in a $\mathbf{C}$-proof from $\Gamma \cap \mathcal{W}_{p}^{+}$, an element of $\Gamma \cap \mathcal{D}_{p}$ or a result of applying the rules TRANS, or UG to preceding lines of the proof. Where $\Gamma \cup\{A\} \in \mathcal{W}_{p}^{+} \cup \mathcal{D}_{o}$, define $\vdash_{\mathbf{C} *}$ by $\Gamma \vdash_{\mathbf{C} *} A$ iff there is a $\mathbf{C} *$-proof for $A$ from $\Gamma$. Obviously, with this definition, for every $\Gamma \cup\{A\} \subseteq \mathcal{W}_{p}, \Gamma \vdash_{\mathbf{C}} A$ iff $\Gamma \vdash_{\mathbf{C} *} A$, but remark that e.g. $\llbracket P a \rrbracket \vdash_{\mathbf{C} *}+\neg \exists x P x$, whereas evidently $P a \nvdash_{\mathbf{C}}+\neg \exists x \neg P x$ and $P a \nvdash_{\mathbf{C} *}+\neg \exists x \neg P x$.

Suppose $\Gamma \nvdash_{\mathbf{C}} A$, where $\Gamma \cup A \subseteq \mathcal{W}^{+}$, whence $\Gamma \nvdash_{\mathbf{C} *} A$. Let $\left\langle B_{1}, B_{2}, \ldots\right\rangle$ be an enumeration of all elements of $\mathcal{W}_{p}^{+} \cup\left\{\llbracket A, C \rrbracket \mid C \in \mathcal{W}_{p}^{+}\right\}$such that if $B_{i}=$ $\exists \beta C(\beta)$ then $B_{i+1}=C(\alpha)$ and if $B_{i}=\llbracket A, \exists \beta C(\beta) \rrbracket$ then $B_{i+1}=\llbracket A, C(\alpha) \rrbracket$, where $\alpha \in \mathcal{O}^{\prime}$ does not occur in $\left\{B_{1}, \ldots, B_{i}\right\}$. Define:

$$
\begin{aligned}
& \Delta_{0}=C n_{\mathbf{C}}(\Gamma) \\
& \begin{cases}\Delta_{i+1}=C n_{\mathbf{C} *}\left(\Delta_{i} \cup\left\{B_{i+1}\right\}\right) & \text { if } A \notin C n_{\mathbf{C} *}\left(\Delta_{i} \cup\left\{\llbracket B_{i+1} \rrbracket\right\}\right) \\
\Delta_{i+1}=\Delta_{i} & \text { otherwise }\end{cases} \\
& \Delta=\Delta_{0} \cup \Delta_{1} \cup \ldots
\end{aligned}
$$

We show that $\Delta$ has the following properties:

1. $\Gamma \subseteq \Delta$. Immediately.

2. $A \notin \Delta$. Immediately.

3. $\Delta$ is deductively closed. Immediately.

4. $\Delta$ is $\omega$-complete i.e. if $\exists \alpha A(\alpha) \in \Delta$ then $A(\beta) \in \Delta$ for at least one $\beta \in \mathcal{C} \cup \mathcal{O}$. Suppose $\exists \alpha A(\alpha) \in \Delta, B_{i}=\exists \alpha A(\alpha)$ and there is no $\beta \in \mathcal{C} \cup \mathcal{O}$ such that $A(\beta) \in \Delta$. But then also $\Delta_{i} \cup\left\{B_{i+1}\right\} \vdash_{\mathbf{C}} A$, with $B_{i+1}=A(\beta)$ for some $\beta \in \mathcal{O}$ that does not occur in $\Delta_{i}$. Hence, in view of Lemma $2, \Delta_{i} \vdash_{\mathbf{C}} A$ or $\Delta_{i} \vdash_{\mathbf{C}} \llbracket+* A(\beta), A \rrbracket$. The former disjunct is impossible in view of property 
2 and the latter disjunct entails $\Delta_{i} \vdash_{\mathbf{C}} \llbracket+\neg \exists \alpha A(\alpha), A \rrbracket$ ( $\beta$ does not occur in $\Delta_{i} \cup\{A\}$ ), whence $\Delta_{i} \vdash \llbracket A \rrbracket$ (by $\Delta_{i} \vdash_{\mathbf{C}} \llbracket \exists \alpha A(\alpha) \rrbracket$ and TRANS), which is also impossible in view of property 2 .

5. $\Delta$ is $\omega *$-complete i.e. if $\llbracket A, \exists \alpha C(\alpha) \rrbracket \in \Delta$ then $\llbracket A, C(\beta) \rrbracket \in \Delta$ for at least one $\beta \in \mathcal{C} \cup \mathcal{O}$. Suppose $\llbracket A, \exists \alpha C(\alpha) \rrbracket \in \Delta, B_{i}=\llbracket \exists \alpha C(\alpha) \rrbracket$ and there is no $\beta \in \mathcal{C} \cup \mathcal{O}$ such that $C(\beta) \in \Delta$. But then also $\Delta_{i} \cup\left\{B_{i+1}\right\} \vdash_{\mathbf{C} *} A$, with $B_{i+1}=\llbracket A, C(\beta) \rrbracket$ for some $\beta \in \mathcal{O}$ that does not occur in $\Delta_{i}$. Hence, in view of $\Delta_{i} \nvdash_{\mathbf{C} *} A, \Delta_{i} \cup \llbracket C(\beta) \rrbracket \vdash_{\mathbf{C}_{*}} A$ and therefore $\Delta_{i} \cup \llbracket \exists \alpha A(\alpha), A \rrbracket \vdash_{\mathbf{C}} A$ (consider a proof that contains a line $j$ saying $\llbracket A, \exists \alpha C(\alpha) \rrbracket$. We can add a line $\llbracket C(\beta),+* C(\beta) \rrbracket$. Because $\Delta_{i} \cup \llbracket C(\beta) \rrbracket \vdash_{\mathrm{C} *} A$, we can add a number of lines resulting in $\llbracket A,+* C(\beta) \rrbracket$ and therefore also $\llbracket A,+\neg \exists \alpha C(\alpha) \rrbracket$ can be added, which with line $j$ allows us to conclude $\llbracket A \rrbracket$.) This would entail $\Delta \cup \llbracket \exists \alpha A(\alpha), A \rrbracket \vdash_{\mathbf{C} *} A$ and hence also $\Delta \vdash_{\mathbf{C} *} A$, which is impossible in view of property 2 .

6. For every $C \in \mathcal{W}_{o}^{+}$holds that if $C \notin \Delta$, then $\ddagger C \in \Delta$. Suppose $C \notin \Delta$ and $\ddagger C \notin \Delta$. Then there are $i, j \in \mathbb{N}$ such that $\Delta_{i} \cup\{C\} \vdash_{\mathbf{C}} A$ and $\Delta_{j} \cup\{\ddagger C\} \vdash_{\mathbf{C}}$ $A$. But then, by Corollary 3 , also $\Delta_{i} \cup \Delta_{j} \vdash_{\mathrm{C}} A$, which is impossible in view of property 2 .

Where $\alpha \in \mathcal{C} \cup \mathcal{O}^{\prime}$, let $\bar{\alpha}=\{\alpha\} \cup\{\beta \mid \alpha=\beta \in \Delta\}$. Observe that the set $\left\{\bar{\alpha} \mid \alpha \in \mathcal{C} \cup \mathcal{O}^{\prime}\right\}$ is a partition of the set $\mathcal{C} \cup \mathcal{O}^{\prime}$.

Define $M$ as the triple $\langle v, w, D\rangle$, where $D=\{\bar{\alpha} \mid \alpha \in \mathcal{C} \cup \mathcal{O}\}, v$ is defined by:

1. for every $\alpha \in \mathcal{C} \cup \mathcal{O}: v(\alpha)=\bar{\alpha}$,

2. $v(\sigma)=1$ iff $\sigma \in \Delta$,

3. $v\left(\sigma_{\neg}\right)=1$ iff $\neg \sigma \in \Delta$,

4. for every $\pi^{r} \in \mathcal{P}^{r}: v\left(\pi^{r}\right)=\left\{\left\langle v\left(\alpha_{1}\right), \ldots, v\left(\alpha_{r}\right)\right\rangle \mid \pi \alpha_{1} \ldots \alpha_{r} \in \Delta\right\}$,

5. for every $\pi^{r} \in \mathcal{P}^{r}: v\left(\pi_{\neg}^{r}\right)=\left\{\left\langle v\left(\alpha_{1}\right), \ldots, v\left(\alpha_{r}\right)\right\rangle \mid \neg \pi \alpha_{1} \ldots \alpha_{r} \in \Delta\right\}$,

6. $v(\neg \cdot=\cdot)=\{\langle v(\alpha), v(\beta)\rangle \mid \neg \alpha=\beta \in \Delta\}$,

7. $v(+\neg \cdot=\cdot)=\{\langle v(\alpha), v(\beta)\rangle \mid+\neg \alpha=\beta \in \Delta\}$, and

8. $v(\cdot=\cdot)=\{\langle v(\alpha), v(\beta)\rangle \mid \alpha=\beta \in \Delta\}$

and $w: \mathcal{W}_{o}^{+} \mapsto\{0,1\}$ is defined by

1. for every $B \in \mathcal{W}_{o}: w(B)=1$ iff $B \in \Delta$, and

2. for every $B \in \mathcal{W}_{o}: w(+B)=1$ iff $+B \in \Delta$.

Note that, for all $B \in \mathbb{P}_{o} \cup\{A \mid \alpha, \beta \in \mathcal{C} \cup \mathcal{O} ; A=\alpha=\beta$ or $A=+\neg \alpha=\beta$ or $A=+\neg \alpha=\beta\}$,

$$
I_{v}(B) \text { iff } B \in \Delta \text {. }
$$

For all $B \in \mathcal{W}_{o}$, if $v_{M}(B)=1$ then $\left(w(B)=1\right.$ or $\left.I_{v}(B)\right)$ and therefore $B \in \Delta$. We obtain,

$$
\text { if } B \in \mathcal{W}_{o} \text { and } v_{M}(B)=1 \text { then } B \in \Delta \text {. }
$$

For all $B \in \mathcal{W}_{o}$, if $B \in \Delta$ then $w(B)=1$ and, when $B$ is of the form $\neg \alpha=\beta$, $I_{v}(B)$. Consequently, $B \in \Delta$. We obtain,

$$
\text { if } B \in \mathcal{W}_{o} \text { and }+B \in \Delta \text { then } v_{M}(+B)=1 \text {. }
$$


We shall prove that, for all $B \in \mathcal{W}_{o}^{+}$,

$$
v_{M}(B)=1 \text { iff } B \in \Delta .
$$

by means of an induction on the complexity of $B$ (the complexity of $B$ is computed by counting all occurrences of $\neg \neg, \vee$ and $\exists$ ). Note that I omit all statements that immediately follow from (2) or (3).

For the induction basis, we have the following cases.

- $B \in \mathbb{P}$. If $B \in \Delta$ then $w(B)=1$ (5) and by (1), $I_{v}(B)$ (6). (5) and (6) together entail $v_{M}(B)=1$.

- $B=\neg C$ and $C \in \mathbb{P}$. If $\neg C \in \Delta$ then $w(\neg C)$ (7) and, by (1), $I_{v}(\neg C)(8)$. (7) and (8) together entail $v_{M}(\neg C)=1$.

- $B=\alpha=\beta$. If $\alpha=\beta \in \Delta$ then $v(\alpha)=\bar{\alpha}=\bar{\beta}=v(\beta)$ (9). If $\alpha=\beta \in \Delta$ then, by (1), $I_{v}(\alpha=\beta)(10)$. (9) and (10) together entail $v_{M}(\alpha=\beta)=1$.

- $B=\neg \alpha=\beta$. If $\neg \alpha=\beta \in \Delta$ then $w(\neg \alpha=\beta)=1$ (11) and, by (1), $I_{v}(\neg \alpha=\beta)(12)$. If $v(\alpha)=v(\beta)$ then, by (12), $I_{v}(\neg \alpha=\alpha)$ whence $T_{M}(v(\alpha))$ holds. (11) and $\left(v(\alpha) \neq v(\beta)\right.$ or $\left.T_{M}(v(\alpha))\right)$ together entail $v_{M}(\neg \alpha=\beta)=1$.

- $B=+C$ and $C \in \mathbb{P}$. If $\ddagger\left[v_{M}(\neg C)=1\right]$ then $w(\neg C)=0$ or $w(+C)=1$ or $I_{v}(C)$. If $w(\neg C)=0$ then $\neg C \notin \Delta$ whence, by property $6,+C \in \Delta$. If $w(+C)=1$ then $+C \in \Delta$. If $I_{v}(C)$ then, by (1), $C \in \Delta$ whence, by $C \vdash_{\mathrm{C}}+C,+C \in \Delta$.

- $B=+\neg C$ and $C \in \mathbb{P}$. If $\ddagger\left[v_{M}(C)=1\right]$ then $w(C)=0$ or $w(+\neg C)=1$ or not $I_{v}(C)$ or $I_{v}(\neg C)$. If $w(C)=0$ then $C \notin \Delta$ whence, by property 6 , $+\neg C \in \Delta$. If $w(+\neg C)=1$ then $+\neg C \in \Delta$. If $I_{v}(\neg C)$ then, by (1), $\neg C \in \Delta$ and, by $\neg C \vdash_{\mathrm{C}}+\neg C$, also $+\neg C \in \Delta$. Finally, if not $I_{v}(C)$ then, by (1), $C \notin \Delta$ whence, by property $6,+\neg C \in \Delta$.

- $B=+\alpha=\beta$. If $\ddagger\left[v_{M}(\neg \alpha=\beta)=1\right]$ then $w(\neg \alpha=\beta)=0$ or $w(+\alpha=\beta)=1$ or $v(\alpha)=v(\beta)$. If $w(\neg \alpha=\beta)=0$ then $\neg \alpha=\beta \notin \Delta$ whence, by property 6 , $+\alpha=\beta \in \Delta$. If $w(+\alpha=\beta)=1$ then $+\alpha=\beta \in \Delta$. Finally, if $v(\alpha)=v(\beta)$ then also $\alpha=\beta \in \Delta$ or $\alpha=\beta$. In both cases $+\alpha=\beta \in \Delta$.

- $B=+\neg \alpha=\beta$. If $\ddagger\left[v_{M}(\alpha=\beta)=1\right]$ then not $I_{v}(\alpha=\beta)$ or $I_{v}(+\neg \alpha=\beta)$ or $v(\alpha) \neq v(\beta)$ or $T_{M}(v(\alpha))$. If not $I_{v}(\alpha=\beta)$ then $\alpha=\beta \notin \Delta$ whence, by property $6,+\neg \alpha=\beta \in \Delta$. If $I_{v}(+\neg \alpha=\beta)$ then, by (1) $+\alpha=\beta \in \Delta$. Either $v(\alpha) \neq v(\beta)$ or $v(\alpha)=v(\beta)$. If $v(\alpha) \neq v(\beta)$ then $\alpha=\beta \notin \Delta$ whence, by property $6,+\neg \alpha=\beta \in \Delta$. Suppose now $v(\alpha)=v(\beta)$ and $T_{M}(v(\alpha))$. Then either $I_{v}(\neg \alpha=\alpha)$ and therefore $I_{v}(\neg \alpha=\beta)$ whence, by $1, \neg \alpha=\beta \in \Delta$ and thus $+\neg \alpha=\beta \in \Delta$, or there is an $r$-ary predicate $\pi$ and $\alpha_{1}, \ldots, \alpha_{r-1} \in D$, such that $\left.\left\langle\alpha_{1}, \ldots, \alpha_{i}, v(\alpha), \alpha_{i+1}, \ldots, \alpha_{r-1}\right\rangle \in v(\pi) \cap v\left(\pi_{\neg}\right)\right)$ '. Hence there is a $C(\alpha) \in \mathbb{P}_{o}$ such that $I_{v}(C(\alpha))$ and $I_{v}(\neg C(\alpha))$, and therefore, by $v(\alpha)=v(\beta)$, also $I_{v}(\neg C(\beta))$ and, by (1), $\{C(\alpha), \neg C(\beta)\} \subset \Delta$. This entails $+\neg \alpha=\beta \in \Delta$ (AS7).

For the induction step, suppose $v_{M}(C)=1$ iff $C \in \Delta$, for every $C$ of complexity lower than $c$. We prove that $v_{M}(B)=1$ iff $B \in \Delta$ for every $B$ of complexity $c$.

Given (2) and (3), we only need to prove that if $B \in \Delta$ then $v_{M}(B)=1$ where $B \in \mathcal{W}_{o}$ and if $\ddagger\left[v_{M}(\ddagger B)=1\right]$ then $B \in \Delta$ where $B=+C$ and $C \in \mathcal{W}_{o}$. 
- $B=D \vee E$. Immediately in view of $D \vee E,+* D \vdash_{\mathbf{C}} E$ and $D \vee E,+* E \vdash_{\mathbf{C}} D$.

- $B=\neg(D \vee E)$. Immediately in view of $\neg(D \vee E) \vdash_{\mathbf{C}} * D$ and $\neg(D \vee E) \vdash_{\mathbf{C}} * E$.

- $B=\exists \alpha D(\alpha)$. Immediately in view of the $\omega$-completeness of $\Delta$.

- $B=\neg \exists \alpha D(\alpha)$. Immediately in view of $\neg \exists \alpha D(\alpha) \vdash_{\mathbf{C}} * D(\beta)$ for all $\beta \in \mathcal{C} \cup \mathcal{O}$.

- $B=+(D \vee E)$. Immediately in view of $+D \vdash_{\mathbf{C}}+(D \vee E)$ and $+E \vdash_{\mathbf{C}}$ $+(D \vee E)$.

- $B=+\neg(D \vee E)$. Immediately in view of $+D,+E \vdash_{\mathbf{C}}+(D \wedge E)$.

- $B=\neg \neg D$. Immediately in view of $\neg \neg D \vdash_{\mathbf{C}} D$.

- $B=+\neg \neg D$. Immediately in view of $+D \vdash_{\mathbf{C}}+\neg \neg D$.

- $B=+\exists \alpha D(\alpha)$. Immediately in view of $D(\beta) \vdash_{\mathbf{C}} \exists \alpha D(\alpha)$ for all $\beta \in \mathcal{C} \cup \mathcal{O}$.

- $B=+\neg \exists \alpha D(\alpha)$. Suppose $\ddagger\left[v_{M}(\ddagger B)=1\right]$ and thus $w(\exists \alpha D(\alpha))=0$ or $w(+\neg \exists \alpha D(\alpha))=1$ or for all $\beta \in \mathcal{C} \cup \mathcal{O}, v_{M}(+* D(\beta))=1$. The two first cases are obvious. In the last case, by the induction hypothesis, $+* D(\beta) \in \Delta$ for all $\beta \in \mathcal{C} \cup \mathcal{O}$ (13). Suppose $+\neg \exists \alpha D(\alpha) \notin \Delta$. Hence there is an $i$ such that $\Delta_{i} \cup\{+\neg \exists \alpha D(\alpha)\} \vdash_{\mathbf{C}} A$ whence, in view of Lemma $2, \Delta_{i} \vdash_{\mathbf{C}} \llbracket A, \exists \alpha D(\alpha) \rrbracket$ and therefore $\llbracket A, \exists \alpha D(\alpha) \rrbracket \in \Delta$. In view of the $\omega *$-completeness of $\Delta$ there exists a $\gamma$ such that $\llbracket A, D(\gamma) \rrbracket \in \Delta$. As a consequence there is a, $\Delta_{i} \cup\{+*$ $D(\gamma)\} \vdash_{\mathbf{C}} A$ whence $+* D(\gamma) \notin \Delta$, which contradicts (13).

By (4) and properties 1 and 2 , we obtain $\Gamma \nvdash_{\mathbf{C}} A$.

\section{Sketch of the proof of Theorem 9}

Suppose

$$
\Gamma \nvdash_{\mathbf{C}}+A
$$

where $\Gamma \cup+A \subseteq \mathcal{W}^{+}$and

there is some $C$ such that $\Gamma \nvdash_{\mathbf{C L}} C$.

The latter supposition entails

$$
\Gamma \nvdash_{\mathbf{C}}+\exists(D \wedge \neg D) \text { for every } D \in \mathcal{W}_{p},
$$

in view of Lemma 4 .

Let $L=\left\langle B_{1}, B_{2}, \ldots\right\rangle$ be an infinite list of all members of $\mathcal{W}_{p}$ (for the definition of $\mathcal{W}_{p}$, see the previous proof) such that if $B_{i}=\exists \beta C(\beta)$ then $B_{i+1}=C(\alpha)$, where $\alpha \in \mathcal{O}^{\prime}$ does not ocuur in $\Delta_{i}$.

$$
\begin{aligned}
& \Delta_{1}=C n_{\mathbf{C}}(\Gamma) \\
& \Delta_{i+1}=\left\{\begin{aligned}
C n_{\mathbf{C}}\left(\Delta_{i} \cup\left\{B_{i+1}\right\}\right) & \text { if } \Delta_{i} \cup\left\{B_{i+1}\right\} \nvdash_{\mathbf{C}}+A \text { and there is } \\
& \text { no } C \in \mathcal{W}_{p} \text { such that } \\
& \Delta_{i} \cup\left\{B_{i+1}\right\} \vdash_{\mathbf{C}}+\exists(C \wedge \neg C) \\
C n_{\mathbf{C}}\left(\Delta_{i} \cup\left\{\ddagger B_{i+1}\right\}\right) & \text { otherwise }
\end{aligned}\right. \\
& \Delta=\Delta_{1} \cup \Delta_{2} \cup \ldots
\end{aligned}
$$


We prove by means of mathematical induction that, for every $i>1$,

$+A \notin \Delta_{i}$ and there is no $D \in \mathcal{F}$ such that $+\exists(D \wedge \neg D) \in \Delta_{i}$.

For the basic case, $+A \notin \Delta_{1}$ (from supposition (14)) and there is no $D \in \mathcal{W}_{p}$ such that $+\exists(D \wedge \neg D) \in \Delta_{1}$ (from $\left.(16)\right)$.

For the induction step we need to prove that if " $+A \notin \Delta_{i}$ and there is no $D \in \mathcal{W}_{p}$ such that $+\exists(D \wedge \neg D) \in \Delta_{i}$ ", then " $+A \notin \Delta_{i+1}$ and there is no $D \in \mathcal{W}_{p}$ such that $+\exists(D \wedge \neg D) \in \Delta_{i+1}$ ". Suppose that the antecedent holds. There are two cases.

Case 1: (i) $\Delta_{i} \cup\left\{B_{i+1}\right\} \nvdash_{\mathbf{C}}+A$ and (ii) there is no $C \in \mathcal{F}_{p}$ such that $\Delta_{i} \cup$ $\left\{B_{i+1}\right\} \vdash_{\mathbf{C}}+\exists(C \wedge \neg C)$. In this case $\Delta_{i+1}=C n_{\mathbf{C}}\left(\Delta_{i} \cup\left\{B_{i+1}\right\}\right)$ whence $+A \notin \Delta_{i+1}$ (from (i)) and there is no $D \in \mathcal{W}_{p}$ such that $+\exists(D \wedge \neg D) \in$ $\Delta_{i+1}$ (from (ii)).

Case 2: $\Delta_{i} \cup\left\{B_{i+1}\right\} \vdash_{\mathbf{C}}+A$ or there is a $C \in \mathcal{F}$ such that $\Delta_{i} \cup\left\{B_{i+1}\right\} \vdash_{\mathbf{C}}$ $+\exists(C \wedge \neg C)$. In this case $\Delta_{i+1}=C n_{\mathbf{C}}\left(\Delta_{i} \cup\left\{\ddagger B_{i+1}\right\}\right)$ and there is a $C \in \mathcal{F}$ such that

$$
\Delta_{i} \cup\left\{B_{i+1}\right\} \vDash_{\mathbf{C}}+A \sqcup+\exists(C \wedge \neg C) .
$$

Suppose now $+A \in \Delta_{i+1}$ or there is a $D \in \mathcal{W}_{p}$ such that $+D \in \Delta_{i+1}$ and $+\neg D \in \Delta_{i+1}$. But then there would also be a $D \in \mathcal{W}_{p}$, such that (by $+A \vdash_{\mathbf{C}}+(A \vee \exists(D \wedge \neg D))$ and $\left.+D,+\neg D \vdash_{\mathbf{C}}+(A \vee \exists(D \wedge \neg D))\right)$

$$
\Delta_{i} \cup\left\{\ddagger B_{i+1}\right\} \vDash_{\mathbf{C}}+A \sqcup+\exists(D \wedge \neg D) .
$$

From (21) and (22), Corollary 3 warrants that

$$
\Delta_{i} \vDash_{\mathbf{C}}+A \sqcup+\exists(D \wedge \neg D) \sqcup+\exists(C \wedge \neg C) .
$$

This entails (by Lemma 5)

$$
\begin{gathered}
\Delta_{i} \vDash_{\mathbf{C}}+A \text { or } \Delta_{i} \vDash_{\mathbf{C}}+\exists(D \wedge \neg D) \text { or } \\
\Delta_{i} \vDash_{\mathbf{C}}+\exists(C \wedge \neg C) \text { or } \\
\Delta_{i} \cup\{+\neg \exists(C \wedge \neg C),+\neg \exists(D \wedge \neg D)\} \vDash_{\mathbf{C}} A .
\end{gathered}
$$

As $\vdash_{\mathbf{C}}$ is complete with respect to its semantics and as $+\neg \exists(E \wedge \neg E) \in$ $\Delta_{i}$ for every $E \in \mathcal{F}$ (the reader can check that $\vDash_{\mathbf{C}}+\neg \exists(E \wedge \neg E) \in \Delta_{i}$ ), this is in contradiction with the induction hypothesis. Consequently, $+A \notin \Delta_{i+1}$ and there is no $D \in \mathcal{F}$ such that $+\exists(D \wedge \neg D) \in \Delta_{i+1}$.

Now we have

$$
A \notin \Delta,
$$

there is no $D \in \mathcal{W}_{p}$ such that $+D,+\neg D \in \Delta$,

(otherwise there would be an $i$ such that $\left.+\exists(D \wedge \neg D) \in \Delta_{i}\right)$ ) and

$$
\text { for every } D \in \mathcal{W}_{p}, D \in \Delta \text { or } \ddagger D \in \Delta \text {. }
$$


(26) and (27) together entail

$$
\text { for every } D \in \mathcal{W}_{p} \text {, if }+D \in \Delta \text { then } \ddagger D \in \Delta \text {. }
$$

Consequently (remember that $\left.\vdash_{\mathbf{C}}+(D \vee \neg D)\right) D \vee \neg D \in \Delta$, for every $D \in \Delta$, whence, by Theorem 7 item 5 and the fact that $\Delta$ is $\mathbf{C}$-deductively closed,

$$
C n_{\mathbf{C L}}\left(\Delta \cap \mathcal{W}_{p}\right)=\Delta \cap \mathcal{W}_{p}
$$

Using (25-29) one can show by means of the usual methods that $\Delta \cap \mathcal{W}_{p}$ is a CL-model set (i.e. it is maximally non-trivial, CL-deductively closed and $\omega$ complete). Hence there exists a $\mathbf{C L}$-model such that, for all $A \in \mathcal{W}, M \models A$ iff $A \in \Delta$. In view of (25), we finally obtain

$$
\Gamma \nvdash_{\mathbf{C L}} A .
$$

